\title{
Acknowledgement to Reviewers of Challenges in 2018
}

\author{
Challenges Editorial Office
}

MDPI, St. Alban-Anlage 66, 4052 Basel, Switzerland

Published: 10 January 2019

Rigorous peer-review is the corner-stone of high-quality academic publishing. The editorial team greatly appreciates the reviewers who contributed their knowledge and expertise to the journal's editorial process over the past 12 months. In 2018, a total of 34 papers were published in the journal, with a median time to first decision of 23 days and a median time to publication of 43 days. The editors would like to express their sincere gratitude to the following reviewers for their cooperation and dedication in 2018:

\begin{tabular}{ll} 
Adamczyk, Janusz & Fistola, Romano \\
Ahel, Ivan & Fonchingong, Charles Che \\
Andrae, Anders S. G. & Gagné, Isaac \\
Andrew, Carrie & Gassman, Natalie R. \\
Arbuthnott, Katherine & Ghiani, Emilio \\
Asdrubali, Francesco & Glaeser, Jessie \\
Assari, Shervin & Gonzalez, Luis Sanchez \\
Bachor, Vernon & Graham, Hilary \\
Bailey, Catherine & Gray, Ben \\
Barton, David C. & Graziani, Grazia \\
Bastante-Ceca, Maria Jose & Hajiloo, Hamzeh \\
Bekele, Dawit & Harris, Leanne \\
Beneke, Sascha & Harvey, Inman \\
Bermejo-Nogales, Azucena & Haseltine, Cynthia \\
Bevilacqua, Carmelina & Hekkenberg, Robert \\
Bishop, Laura & Huynen, Maud \\
Board, Mary & Irwin, Jennifer \\
Brough, Mark & James, Katherine A. \\
Brown, Mary Jean & Johansen, Guro Gravem \\
Busby, Joshua W. & Jurdak, Raja \\
Butler, Colin D & Katina, Polinpapilinho F. \\
Capitman, John & Kauffman, Kathryn Marie \\
Cerchione, Roberto & Keida, Elizabeth \\
Chen, Chong & Keynan, Irit \\
Comas-Garcia, Andreu & Khorasani, Negar Elhami \\
Correa, Patricia Isabel Matus & Kirk, Sara \\
Craig, Jeffrey & Klaessig, Frederick \\
Dettori, Marco & Koh, David \\
Di Maro, Antimo & Konstantakopoulou, Foteini \\
Dimopoulos, Konstantinos & Korajkic, Asja \\
Elhoseny, Mohamed & Kyamakya, Kyandoghere \\
Esmaeili, Behzad & Laverack, Glenn \\
Faraone Mennella, Maria Rosaria & Leonti, Marco \\
Fernández-Caramés, Tiago M. & Leung, Anthony K.L. \\
Li, Lijuan & Rikard, Robert Vann \\
\hline & \\
\hline &
\end{tabular}


Logan, Alan C.

Lowry, Christopher

Macioce, Fabio

Marino, Davide

Martino, David J.

Mascaro, Jennifer

Matheson, Anna

Mcgrath, Cormac

McGrattan, Kevin B.

Melo, Pedro

Motoyama, Keiichi

Myers, Samuel

Nash, Carol

Nieber, Karen

Niemann, Jorg

Nurunnabi, Mohammad

Oztanriseven, Furkan

Palazzo, Luca

Palumbo, Elisabetta

Parker, Judith

Pavón García, Ignacio

Pepi, Milva

Poirier, Guy G.

Pollitt, Krystal

Poltronieri, Palmiro

Poncet, Sébastien

Pratley, Pierre

Prescott, Susan

Rezzani, Rita
Ross, Adam

Rossi, Franca

Runstadler, Jonathan A.

Sahoo, Kamalakanta

Sarathy, Vanessa V.

Schweiger, Martin

Slama, James T.

Smith, Jonathan W.

Smith, Eric

Soderquist, Klas Eric

Sørensen, Kristine

Stauvermann, Peter Josef

Stewart, Alex G.

Svendsen, Morten Bo Søndergaard

Tengland, Per-Anders

Thomas, Evan

Timinszky, Gyula

Tong, Jingjing

Tsai, Wen-Hsien

Turner, Jonathan

Virag, Laszlo

Visconti, Guido

Warren, Joshua

$\mathrm{Wu}, \mathrm{We}-\mathrm{Tao}$

$\mathrm{Wu}$, Jianyong

Zambon, Ilaria

Zamyatnin, Jr., Andrey A.

Zinszer, Kate

(C) 2019 by the authors. Licensee MDPI, Basel, Switzerland. This article is an open access article distributed under the terms and conditions of the Creative Commons Attribution (CC BY) license (http://creativecommons.org/licenses/by/4.0/). 